\title{
Aprendizaje emocional para convivencia, rendimiento y motivación: una muestra de estudio en educación preescolar y primaria
}

Enviado: 21 de abril de 2021 / Aceptado: 11 de junio de 2021 / Publicado: 12 de julio de 2021

CARMEN LUCÍA ZÚÑIGA VILLEGAS

UNINI-MX Universidad Internacional Iberoamericana carmen.zuniga1@doctorado.unini.edu.mx

MARÍA JESÚS LUQUE ROJAS

Universidad de Málaga, España mjluquerojas@uma.es

\section{RESUMEN}

El propósito del estudio es implementar una propuesta pedagógica a través del juego, la recreación y la lúdica para mejorar el aprendizaje emocional en pro de la convivencia escolar en los niños y niñas del nivel de preescolar y básica primaria en la Institución Educativa Pacarní del municipio de Tesalia-Huila, con una metodología mixta y un diseño por fases y alcance descriptivo, en una muestra intencional de 25 niños y niñas de los grados preescolar y primero de primaria, al conjunto muestral se aplican una encuesta, revisión documental y entrevista semiestructurada, en tanto que la implementación y su impacto se evalúan a través de observación, los datos serán procesados por estadística en sus datos cuantitativas y por categorías emergentes a partir de las categorías de análisis para los datos cualitativos.

Los resultados diagnósticos indicaron por causalidades la no aceptación familiar, el bajo autoconcepto y

\section{ABSTRACT}

Emotional learning for coexistence, performance and motivation: a sample study in preschool and primary education

The purpose of the study is to implement a pedagogical proposal through games, recreation and play to improve emotional learning in favor of school coexistence in children at the preschool and elementary school level in the Pacarní Educational Institution of the municipality of Tesalia - Huila, with a mixed methodology and a design by phases and descriptive scope, in an intentional sample of 25 boys and girls from preschool and first grade of primary school, a survey, documentary review and semi-structured interview are applied to the sample set, in As long as the implementation and its impact are evaluated through observation, the data will be processed statistically in its quantitative data and by emerging categories from the analysis categories for qualitative data. 
el rechazo de compañeros, las problemáticas (maltrato a compañeros, conductas disruptivas, indisciplina, autoritarismo, oposicionismo, burlas, palabras soeces y aislamiento), en tanto que como fortalezas se halló amistad y dedicación. En tanto que, los resultados posteriores a la estrategia aplicada se segmentan así: para Actividades Lúdicas (participación, no partícipes, responsabilidad y desaliento), para proceso de aprendizaje, motivación y desempeño escolar (anomía, interés, orientación) y para la convivencia sobre persistencia de fallas (individualismo, apodos, rechazo, imposición de opinión, división entre niños y niñas) y como convivencia positiva (participan, comunican sus cosas, compañerismo, receptividad).

Palabras Clave: juego, aprendizaje, convivencia, desempeño estudiante, primera infancia.
The diagnostic results indicated by causalities family non-acceptance, low self-concept and peer rejection, problems (maltreatment of peers, disruptive behaviors, indiscipline, authoritarianism, oppositionism, teasing, profanity and isolation), while strengths he found friendship and dedication. Whereas, the results after the applied strategy are segmented as follows: for Playful Activities (participation, non-participants, responsibility and discouragement), for the learning process, motivation and school performance (anomie, interest, orientation) and for coexistence on persistence of failures (individualism, nicknames, rejection, imposition of opinion, division between boys and girls) and as positive coexistence (they participate, communicate their things, companionship, receptivity).

Keywords: play, learning, coexistence, student performance, early childhood.

\section{INTRODUCCIÓN}

La institución educativa acumula los denominados documentos observadores de estudiantes, escritos que contienen en su mayoría llamados de atención por el uso de vocabulario pesado, agresiones físicas y por situaciones de conflicto en clase y en horas de recreo. De otra parte, continuamente se realizan citaciones a padres de familia para solucionar situaciones de mal comportamiento e irrespeto, falta de seguimiento de normas e instrucciones y casos del llamado matoneo. En algunas ocasiones, se ha encontrado que los estudiantes llevan en sus maletas escolares armas para defenderse o amenazar e intimidar a sus compañeros.

En la base de estas dificultades yacen según la Organización de las Naciones Unidas para la Educación, la ciencia y la cultura (UNESCO) (2011) los conflictos familiares, la absorción del trabajo paterno, la falta de motivación y preparación que existe entre los padres lo que impiden que haya un buen rendimiento escolar y una sana convivencia en las aulas, a ello se suman los fuertes episodios de violencia que ha sufrido el país, las divisiones sociales, la intolerancia y los prejuicios que conducen a la guerra. 
Una atmosfera que se ha convertido en un ambiente envolvente que atrapa a los ciudadanos y que se hace natural a los ojos de los demás ciudadanos en el país, una desensibilización de los sucesos desagradable de la vida y la convivencia con la aparición de una permisividad social de estos eventos como una aceptación soterrada, la cual se agudiza con el hecho de convertir las descritas pautas de coexistencia en trasmisibles de generación en generación. En este sentido, los actuales pobladores las recibieron de sus progenitores y ahora sin mayor dificultad dejan que aparezcan en sus hijos e hijas como una herencia cultural sobre la cual les es esquivo el ejercicio de conciencia en cuanto a lo adverso que representa.

Por tanto, es de vital importancia que la escuela tome como bandera de desarrollo formativo integral y el rol socializador dentro de la especie la ocupación de trabajo hacia la pacificación de las formas de convivencia desde las edades más temprana, a través de la formulación de estrategias las cuales permitan mejorar el ambiente escolar y que se consoliden como un aprendizaje fuerte a instaurar en la cultura de la localidad.

Para Nóbrega y Franco (2014), es importante el trabajo educativo de esta índole desde el preescolar que haga más seguro la adquisición de la competencia emocional como se evidencia en su trabajo "Inteligencia emocional: la comprensión emocional y las relaciones entre pares en el jardín de infancia”. En este, hallaron que la relación entre el incremento de la edad y la Comprensión Emocional CE es directamente proporcional, como constructo en desarrollo continuo; sobre la elección de pares se indica la afinidad emocional e incrementa el nivel de aceptación, en tanto, para las niñas cuenta la popularidad y el liderazgo, pero, es afirmativo que la comprensión emocional influencia las relaciones entre pares. En este sentido, investigadores como Marchant, Milicic y Álamos (2013) corroboran el impacto positivo de la educación emocional en niños en la primera infancia.

En Colombia se encuentra una menor cantidad de material sobre este tipo de literatura investigativa, uno de los hallazgos con mayor similitud fue presentado en universidad española y llevada a cabo en Boyacá denominada "Relación entre Inteligencia emocional y autoestima en niños de 5 años" de Martínez (2015), sobre inteligencia emocional con el apoyo de talleres tanto lúdico-pedagógicos como creativos, en la organización de Desarrollo Infantil, Jorge Eliecer Gaitán, de Tunja, Boyacá.

Los resultados parten de describir la fase diagnóstica donde el 74\% registraron alteraciones emocionales de normal a normal bajo, normal bajo, nivel bajo y nivel inferior, además, el $65 \%$ estuvieron entre autoestima muy baja y autoestima baja; se implementan las actividades 
del programa diseñadas como talleres lúdico-pedagógicos en jornadas activas y creativas de 30 minutos para cuatro meses. Luego, la evaluación final mostró una mejora en los indicadores iniciales.

Un conjunto informativo que lleva al propósito de implementar una propuesta pedagógica a través del juego, la recreación y la lúdica para mejorar el aprendizaje emocional en pro de la convivencia escolar en los niños y niñas del nivel de preescolar y básica primaria en la Institución Educativa Pacarní del municipio de Tesalia-Huila.

\section{Inteligencia y educación emocional}

Gardner (1983) define la inteligencia como la capacidad que se posee para solventar problemas cotidianos, que a su vez permite generar otros problemas nuevos e incluye el crear productos u ofrecer servicios válidos dentro del propio ámbito cultural. Según lo anterior, la mayoría de las personas están en posibilidad de desarrollar todas estas inteligencias hasta poseer en cada una un nivel de competencia razonable a partir de tres factores principales: Dotación biológica, historia de vida personal y los antecedentes culturales e históricos del individuo.

Además, al definir la inteligencia como una capacidad, ese ámbito se convierte en una competencia que consecuentemente se puede enseñar y aprender; esto significa que todos los seres al nacer traen unas potencialidades marcadas, pero, esas potencialidades se van a desarrollar de una manera o de otra dependiendo del medio ambiente, de la cultura y de las experiencias educativas, entre otras.

De otro lado, sobre la inteligencia emocional, como término desde una perspectiva histórica (Carbajal, Flores, Polo y Torres, 2018) se ubica con datación en 1964 mediante artículo de Michael Beldoch, luego aparece de nuevo en 1966 asociada a publicación de B. Leuner dentro de lo correspondiente a temáticas académicas de psiquiatría y psicología infantil.

El término cobra otro matiz con la inclusión del mismo en la teoría de Gardner (1983) que se entiende a modo de destreza la cual tienen las personas para buscar el equilibrio de sus propios sentimientos, de tal manera que se puedan distinguir y rotular adecuadamente sus vivencias emotivas, y después utilizar esta información para precisar una conducta a seguir; además, gracias a esta inteligencia se establece la valoración de las expresiones verbales y no verbales utilizando todo el marco emocional para la construcción de vínculos relacionales y para la resolución de conflictos. 
Pero, la inteligencia emocional es igualmente asumida por Goleman (1995) para darle reconocimiento general y con ello indicar que las personas con inteligencia emocional tienden a manejar un perfil que les hace mostrarse enérgicas, caracterizadas por expresar sus sentimientos sin rodeos, ser positivas en su forma de ver e interpretar la realidad, sumado a que soportan de manera adecuada la tensión y poseen un sentido claro de la vida; lo que se puede concretar en asociar la inteligencia emocional con aquellas personas competentes en reconocer sus emociones, las de los demás y reaccionar coherentemente en torno a ellas.

Desde la inteligencia emocional como capacidad hacia su proceso formativo en los seres humanos, se parte del concepto para la educación emocional según Casel (2017, citado por Hermans y Collins, 2018) quien lo expone así:

el proceso a través del cual los niños y adultos adquieren y aplican efectivamente el conocimiento, las actitudes y las habilidades necesarias para comprender y manejar las emociones, establecer y lograr metas positivas, sentir y mostrar empatía por los demás, establecer y mantener relaciones positivas, y tomar decisiones responsables (p. 2).

Así, la educación emocional implica aprovechar las potencialidades y desarrollar esos haberes previos hacia una transformación de las cogniciones, observables en las actitudes y conductas de un individuo con otros en los escenarios donde se inserta e interactúa. Agregan Cefai y Cavioni (2014), que la educación emocional genera conocimientos paralelo a las destrezas necesarias para la comprensión de sí mismo y para con las otras personas, junto a dar a conocer y regular las emociones; un conjunto de habilidades útiles indispensables en la construcción de relaciones tanto sanas como afectivas, a lo cual se suma el tomar decisiones positivas, de cualidades responsables y éticas, a la par que implementar esas fortalezas y capacidades en la superación de las dificultades presentes normalmente en tareas sociales y académicas.

\section{Regulación emocional en edades tempranas}

Es presentada como las capacidades para manejar emociones de manera positiva y productiva, lo que implica ser conscientes de los sentimientos que se experimentan, monitorear sus intensidades para adelantar su modificación cuando se haga necesario como ayuda para enfrentar las diferentes situaciones del diario vivir y compartir.

Los niños que tienen dificultades en esta área, es decir, para Ayers, Hamada y Wyatt (2011) expresar emociones adecuadamente, pueden no tener los recursos para centrarse en el 
aprendizaje, mientras que los que desarrollan la capacidad de mantener la regulación emocional y aflorar las emociones positivas pueden estar más en función de participar mejor con las tareas del aula.

Además, la regulación de las emociones, así como observable en ellos la positividad emocional, se asocian con efectividad social de los niños preescolares; pero, afirman Ayers, Hamada y Zinsser (2012) esa capacidad de permanecer emocionalmente regulado y articulado con manifestaciones emocionales positivas también se refiere a la evaluación de éxito del maestro escolar de los niños durante su estancia en el jardín de infancia.

De este modo se introducen las competencias emocionales las cuales son importantes tanto para el éxito social y académico en la escuela, ellas son omnipresentes en los intercambios relacionales diarios de los niños y niñas indistintamente de con quien se establece; por ello se tiene que:

\footnotetext{
Si la docente se informa acerca de las características y fases de desarrollo de la inteligencia emocional del niño, y aplica sus conocimientos en la interacción con él, entonces, se estará dando atención a un aspecto relevante el desarrollo del niño con la visión de una educación integral (Ministerio de Educación Nacional, 2014, p. 24).
}

El aprendizaje de las competencias emocionales en edades tempranas se soporta en la lúdica, ya que como lo expone Peña (2013), los juegos como prácticas recreativas en definitiva poseen una alta influencia en los procesos de socialización entre los estudiantes, con estos resultados obtenidos indica que los docentes reconocen que los juegos recreativos, son una herramienta para lograr que los alumnos desarrollen actividades favorables.

Whitebread y Basilio (2012), por su parte plantean que el aprendizaje de lo social debe tener su inicio desde el nivel preescolar, para ello han de utilizarse las actividades lúdicas, ya que son precisamente las jornadas lúdicas las que favorecen que los niños participen, interactúen y se integren con sus compañeros y al tiempo aprender a considerar no solo el sí mismo.

\section{Convivencia escolar y social}

La convivencia según Ministerio de Educación Nacional (2014), hace alusión a las diferentes interrelaciones que surgen entre las personas que comparte un mismo espacio y que debe 
guardar un tratamiento armonioso con los demás. De una manera más concreta la coexistencia es el vivir en compañía, de ahí que se observan las relaciones entre quienes son integrantes de un mismo entorno.

Ahora bien, las pautas de convivencia que se pueden dividir en dos bloques, uno que tiende hacia el individualismo y el segundo cuya formación se perfila más hacia el colectivismo. En este sentido, Temis, Wau y Hughes (2007), escriben que los términos colectivismo y el individualismo se han utilizado para referirse a los sistemas de valores existentes dentro de las comunidades pequeñas y a través de grandes grupos culturales.

Por su parte, Temis, Wau y Hughes (2007), indican la existencia de cuatro valores individualistas (ponderación exclusiva de la elección personal, preponderancia de motivación intrínseca, autoestima desmedida y auto-maximización) que reflejan las creencias de los padres sobre los fundamentales requerimientos que lleven al logro exitoso de los niños o niñas para vivir en la sociedad donde se encuentran enmarcados. Una visión sesgada que conduce al individualismo y se aleja de una convivencia en verdad y entonces como lo explican Sánchez y Quiceno (2016), "surge la falta de solidaridad, el egoísmo y la imposibilidad de desplegar una conducta de empatía como factores que facilitan la aparición de la violencia y la indiferencia social” (p.57).

La convivencia social es una situación de entender que no se trata solo de una persona sino de un yo y muchos otros, que es sin lugar a duda el punto de quiebre a la hora de abordar la temática de convivir, la gran dificultad de evitar que una sola identidad busque prevalecer, por ello:

la convivencia como proceso social ha sido afectada por el creciente individualismo, la politización de las relaciones comunitarias, la visión asistencialista que se propone desde los programas estatales y la falta de cohesión social. En este sentido, el no establecimiento de relaciones de convivencia altera el orden social, al no promover vínculos que permitan a las comunidades crear redes orientadas a satisfacer necesidades comunes y a propiciar condiciones de bienestar psicosocial (Sánchez y Quiceno, 2016, p.57).

\section{METOdOLOGÍA}

El desarrollo de esta actividad de tipo investigativa se enmarca en un enfoque mixto, en consideración a que el objeto de estudio es intersubjetivo, razón que viabiliza la ejecución de análisis que se extraen desde lo cualitativo como cuantitativo, este enfoque según Hernández, Fernández y Baptista (2014). 
El diseño investigativo es secuencial, uno de los concebidos para el enfoque mixto, lo cual permite estructurar fases para el cumplimiento de lo prospectado, en este caso una primera fase cuantitativa descriptiva observable en el proceso diagnóstico (encuestas y revisión documental); luego se organiza una fase cualitativa fenomenológica (entrevistas semiestructuradas) en relación con el acopio amplio de cada uno de los aspectos diagnósticos requeridos de tal forma que sean datos insumo para la construcción ajustada de la propuesta pedagógica y finalmente en la cualificación (observación) del impacto que representó la implementación de la propuesta pedagógica diseñada en respuesta al reconocimiento previo de la realidad que identifica al contexto educativo protagónico.

\section{Población y muestra}

La población estudio, según Secretaria de Educación de Tesalia, se plasma en la tabla 1 de la siguiente manera:

Tabla 1. Población. Fuente: Elaboración propia

\begin{tabular}{cc} 
Grado & No. estudiantes \\
\hline Preescolar & 27 \\
\hline $1^{\circ}$ Primaria (2 aulas) & 42 \\
\hline Total participantes & 69 \\
\hline
\end{tabular}

En lo que al muestreo corresponde se utiliza la técnica de muestreo intencional o por conveniencia. Esta permite desde la experiencia del investigador construir muestras homogéneas que les permita consolidar en los participantes una representación típica de la población general; en este caso, los criterios de selección están definidos para la configuración de dos grupos dadas las características inherentes a las edades y los grados formativos alcanzados, por ello se tendrá un primer grupo de preescolar y un segundo para los de primero de primaria.

Así, se define una muestra compuesta por 8 estudiantes de preescolar y 17 de primero de primaria, para un total de 25 participantes, como se presenta en la tabla 2.

Tabla 2. Muestra. Fuente: Elaboración propia

\begin{tabular}{cc} 
Grado & No. estudiantes \\
\hline Preescolar & 8 \\
\hline $1^{\circ}$ Primaria & 17 \\
\hline $\mathbf{N}$ & 25 \\
\hline
\end{tabular}




\section{RESULTADOS}

El presente apartado se estructura con lo arrojado para la fase diagnóstica donde se articulan encuestas, revisión documental y entrevistas semiestructuradas; una vez concluida la exposición diagnóstica se agrega una breve descripción de la intervención y lo correspondiente al impacto de la estrategia en su implementación derivado de la observación aplicada, este conjunto informativo se expone así:

\section{Diagnóstico}

Los resultados de esta fase se presentan en la figura 1. El diagnóstico se desglosa en causalidad problemáticas y fortalezas, así:

Figura 1. Resultados diagnósticos. Fuente: Elaboración propia

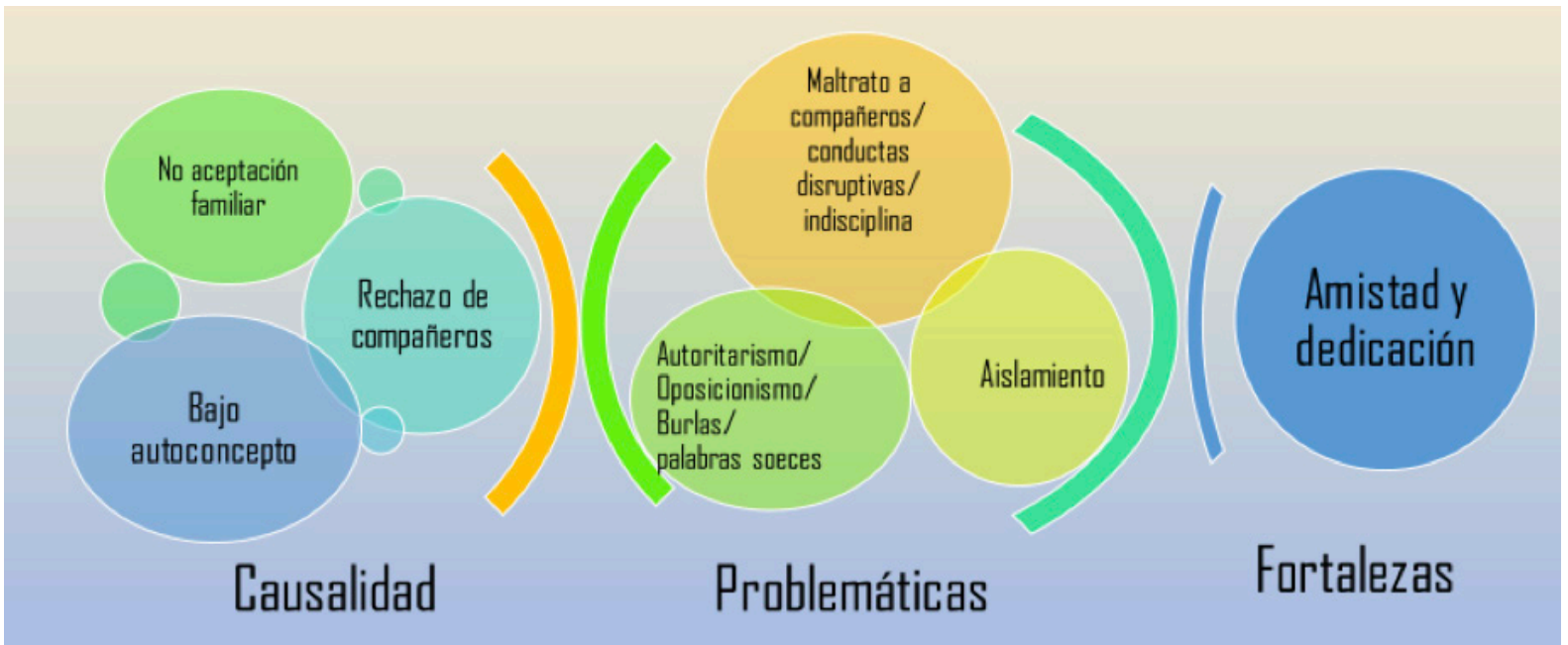

Así, está lo arrojado como causalidades donde se encuentra la percepción por parte del estudiante sobre la no aceptación familiar con la expresión de no encajar en su familia, seguida de un bajo autoconcepto y el rechazo que siente desde sus compañeros durante las jornadas escolares en sus salas de clases y en los otros espacios donde comparte los estudiantes.

Mientras que, las problemáticas que dieron a conocer fueron maltrato a compañeros, conductas disruptivas, indisciplina, autoritarismo, oposicionismo, burlas, palabras soeces y aislamiento; además, está lo referente a las fortalezas, entre las cuales emergen la amistad y dedicación. 


\section{Intervención}

La intervención se diseña y dirige para la primera infancia, en este sentido la base está dada según Bisquerra (2009), y acorde con los resultados de las falencias detectadas, las cuales hacen parte del proceso emocional del Yo mental egoico, en particular tres puntos que se detallan así:

Proceso emocional-Yo mental egoico

- Reflexiona acerca del orgullo y el deseo personal

- Expresa de manera espontánea el amor hacia otras personas, objetos o cosas.

- Reconoce la intimidad y el secreto

Para dar materialización a los tres aprendizajes de inteligencia emocional aquí señalados se estructura un trabajo de un mes que incluye las habilidades que a continuación se citan: Identificación de emociones, Tolerar frustración, Sentir empatía, Control de la ira, Intimidad y secreto, Manejo de la agresividad, Habilidades de cortesía social (2 semanas). Cada semana se trabajan dos de los puntos previamente señalados, a excepción del trabajo de cortesía social que por sus contenidos se extiende a dos semanas. El trabajo de intervención educativa implica un taller lúdico-pedagógico de hora y media para cada aspecto a gestionar como aprendizaje y dentro de la semana se escogen dos días específicos para ello.

\section{Observación posterior a la implementación de estrategia}

La valoración de la estrategia implementada toma varias líneas analíticas la primera de ellas Categoría de análisis Actividades lúdicas emergen las categorías selectivas que a se detallan así: Participación (Participación activa, Atención prestada, Integración), No partícipes (Timidez, Distracción), Responsabilidad (Realizan sus deberes), Desaliento (Desanimo por reglas).

En el caso de la Participación, en alusión a la construcción de lazos de confianza entre los niños para socializar, además que mediante su interés por la actividad lúdica, permite una intermitente curiosidad por esta, acorde con lo anterior, emerge la Participación activa, equivalente a la acción en vía de la actividad y se manifiesta de manera oportuna, se adiciona Atención prestada, consiste en la dedicación que tienen los estudiantes a la hora de indicar los requisitos a una actividad, se agrega la Integración, la cual, se manifiesta en el compartir con el otro y el grupo en su totalidad. 
Después sigue No partícipes, equivalente a los comportamientos o conductas que no integran los grupos de niños, sino que por el contrario logra escindirlos dentro de las actividades lúdicas, por este motivo se manifiesta la Timidez, consiste en la participación de diferentes grupos de actividades con cierto temor e introspección de los estudiantes, en este sentido, la Distracción, se manifiesta con la poca atención que tienen los estudiantes frente a los escenarios, expresado de la siguiente forma: "otros se distraen".

Seguidamente se encuentra Responsabilidad; la cual es una descripción sobre acciones que se ejecutan acorde a los diferentes trabajos, por lo que se desglosa en Realizan sus deberes, de la siguiente manera: "realizan los trabajos con responsabilidad".

Por último, Desaliento, se trata de exponer los estados de ánimo que tienen los estudiantes, a partir de las diferentes imposiciones que da el profesor en la actividad, por ello, se expresa en Desanimo por reglas, es decir, hay una apatía a las normas de las actividades.

Subsecuentemente, se encuentra el efecto en Proceso de aprendizaje, motivación y desempeño escolar, en ella se ubican seis categorías principales con los siguientes soportes: Anomía (Desacato) Interés (Activos, Recursos multimedia, Agrado a la lúdica); Orientación (Receptivos a orientación).

La Anomía asumido como una actitud en contravía a las diferentes normas que presenta el docente a sus estudiantes, de esta manera se sustenta en el Desacato, indicando la actitud oposicionista de los estudiantes al salir del aula y tener actividades por fuera de esta sin acatar las reglas.

Seguidamente se encuentra Interés, reflejándose en la actitud de los estudiantes frente a las diversas estrategias de clase, adicional a esto son proactivos, manifestándose dentro de la subcategoría Activos, trata sobre como los estudiantes asumen las actividades de forma que son propositivos y participativos de estas.

Después de esto, surge los Recursos multimedia, donde se manifiestan las clases con diferentes apoyos que un libro, en este sentido, se expresa de la siguiente forma: “...observar imágenes videos y participar de ellos, cuando se organizan actividades en grupo pequeños participan sin ningún problema”. Por último, se encuentra el Agrado a la lúdica, se expresa el gusto por las actividades que permiten la salida del aula, el juego, la retroalimentación del estudiante con el entorno. 
Finalmente, está la categoría principal Orientación, se dedica a las diferentes sugerencias u normas que se establecen dentro de la clase, a lo cual se sustenta en Receptivos a orientación; donde se demuestra que los estudiantes tienen cierta capacidad de escucha, la cual, se expresa de la siguiente manera: "Al iniciar las actividades escuchan orientaciones y reglas para el desarrollo de las mismas”.

A lo previo se anexa lo referente a la Convivencia, esta tiene ubicadas las siguientes categorías selectivas que se sustentan de la siguiente forma: Fallas de convivencia (Individualismo, Irrespeto, Apodos, Rechazo, Imposición de opinión, División de niños y niñas), y Convivencia positiva (Participan, Comunican sus cosas, Compañerismo, Receptividad).

Las Fallas de convivencia, trata sobre las diferentes conductas que permiten a los niños estar alejados de los otros, o que demuestran no son hábiles para departir junto a los demás, esto se ve reflejado en Individualismo, se trata de la distancia que tienen algunos niños con sus compañeros, además de los pocos tejidos de confianza que se han instalado entre ellos, seguidamente, está Irrespeto, la cual implica diferentes expresiones de burla al manifestar los gustos o intereses, adicional a este, se encuentra Apodos, son representaciones que encuentran los diferentes niños y, a partir de algunos defectos físicos o diversas acciones los caracterizan y ponen sobrenombres.

Además está, Rechazo, donde algunos estudiantes sufren la resistencia de los otros a la hora de compartir o integrar grupos de estudio, es así como se transgrede la violencia en el aula, además, se origina Imposición de opinión, trata sobre la ganancia del debate de cualquier tema agrediendo al otro interlocutor, por último, División de niños y niñas, se trata sobre como los niños tienen algunos grupos de niños y grupos de niñas, es decir, hay una división de los trabajos y actividades en clase que permiten esta división, se expresa de la siguiente manera: "se nota en la organización de grupos los niños aparte de las niñas".

Por último, se encuentra la Convivencia positiva, equivale a los comportamientos que permiten una sana convivencia, es decir, una armonía con el grupo y sus compañeros, lo cual, se manifiesta en Participan se permite una formación conjunta de valores entre el niño y el grupo, agregando a lo anterior, aparece Comunican sus cosas, en el cual, los niños expresan tácitamente los diferentes valores, criterios y se escuchan para que el conocimiento fluya no solo desde la educación, sino desde la experiencia con la otredad, al igual que las otras, se germina el Compañerismo, se trata de integrar a todos los estudiantes que están dentro del curso, en las diversas actividades realizadas, por último, la Receptividad, es decir, la capacidad de entender los errores cometidos y asumir sus consecuencias. 


\section{Consolidación resultados del impacto derivado de la estrategia}

La respuesta de los estudiantes participantes frente a las actividades lúdicas aplicadas fue de cuatro categorías que se observan en la figura dos:

Figura 2. Actividades lúdicas. Fuente: Elaboración propia

\section{ACTIVIDADES LÚDICAS}

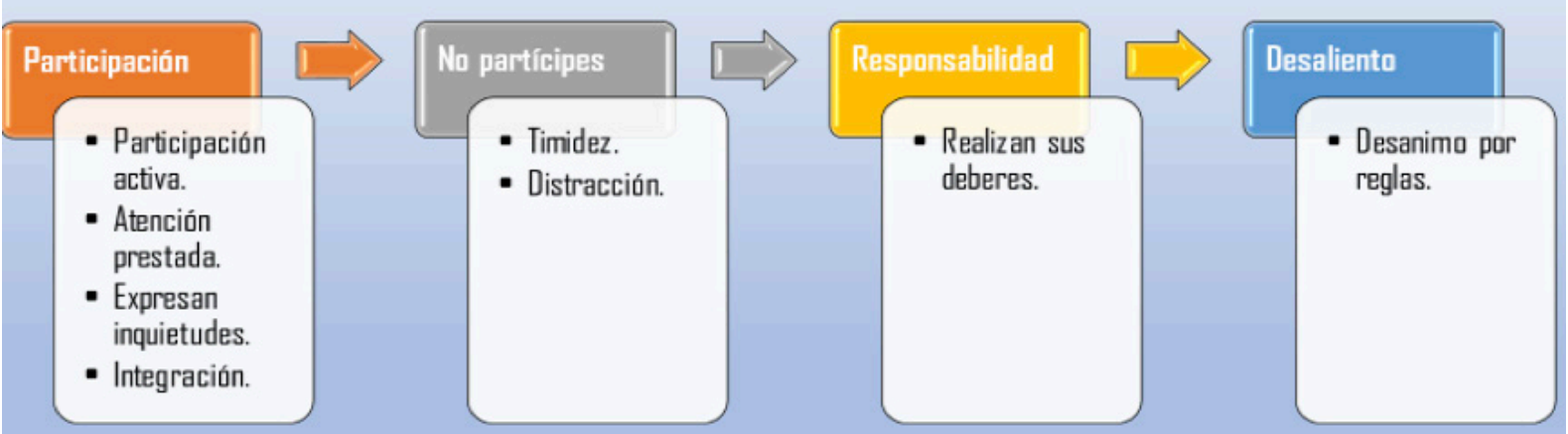

Seguidamente, está el proceso de aprendizaje, motivación y desempeño escolar que se configuró como se exponen en la figura tres así:

Figura 3. Proceso de aprendizaje, motivación y desempeño escolar. Fuente: Elaboración propia

\section{PROCESO DE APRENDIZAJE, MOTIVACIÓN Y DESEMPEÑO ESCOLAR}




El tercer aspecto en análisis fue lo referente a la convivencia, sobre este punto en particular lo arrojado se establece en dos categorías principales y sus correspondientes subcategorías así: Fallas de convivencia (Individualismo, Apodos, Rechazo, Imposición de opinión, División de niños y niñas) junto a Convivencia positiva (Participación, Comunican sus cosas, Compañerismo, Receptividad. Esto se visualiza así en la figura cuatro.

Figura 4. Convivencia. Fuente: Elaboración propia

\section{CONVIVENCIA}

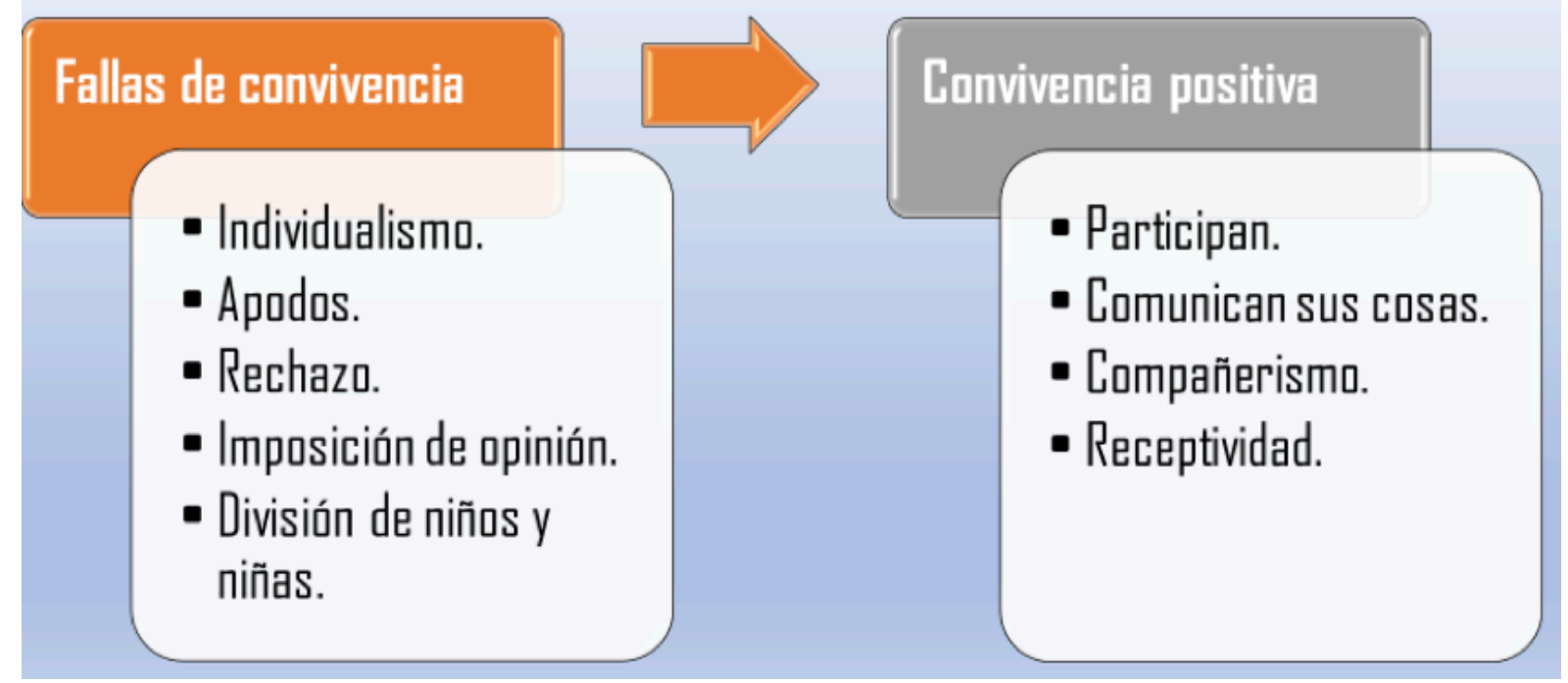

\section{DISCUSIÓN DE RESULTADOS}

El avance académico en el campo de las emociones ha sido un proceso significativo para acrecentar la comprensión de estas, y derivado de ese progreso en el conocimiento, surge la necesidad por hacer de ellas unas convenientes aliadas en la generación del comportamiento humano como producto de una acción con mejor administración sobre las atribuciones emotivas a la información perceptiva, de tal forma que la acción generada sea positiva para ayudar al individuo a desenvolverse mejor en los diferentes escenarios donde se ubica la cotidianidad de las personas.

Entre las actuales acepciones para hacer alusión a las emociones están Carbajal, Flores, Polo y Torres (2018), quienes indican como estas surgen a manera de respuesta dadas por el organismo ante los estímulos que un individuo capta del medio que le rodea, agregan que son procesos propios de la naturaleza humana y están presentes en su cotidianidad, por eso mismo 
el análisis subjetivo de las emociones se obvia en medio de ello y se toma como normalidad de la actuación diaria sin una planeación previa.

La idea de las emociones como irrelevantes dentro de la cotidianidad al profundizar sobre su papel y su desarrollo se transforma con los planteamientos de Gardner (1983) que las postula como una habilidad sobre la cual es necesario formar destreza, ya que ese desempeño se asocia con la capacidad de inteligencia del individuo esencial en la construcción de vínculos interrelaciónales, la solución de conflictos y direccionar de una manera más sana la misma vida.

Precisamente hacia este interés formativo se atiende una población de estudiantes de máximo 8 años de edad inscritos en una institución educativa en zona rural del sur en el departamento del Huila en Colombia, como una intervención pedagógica que contribuya ante problemáticas de maltrato a compañeros, conductas disruptivas, indisciplina, el autoritarismo, actitudes oposicionistas, así como una variada gama de actuaciones de burla y palabras soeces para con los compañeros y en las actitudes de solitarios.

También se encontró en los estudiantes en relación con el diagnóstico previo factores de causalidad, entre los cuales están el que ellos sienten el no ser una parte aceptada de su familia, esto para Silva y Calvo (2014) tiene que ver con los procesos afectivos de inserción en su grupo social familiar, los cuales no han sido llevados a cabo de manera adecuado para crear una situación de insatisfacción que incide negativamente en el desarrollo emocional de los niños.

Henao y García (2009), exponen que se trata de pensamientos interpretativos de los progenitores cuyo sustrato primordial es la comunicación, manifestaciones de sus figuras paternas en cuanto a aprobación o no de sus acciones básicamente en consideración al cómo se expresan cuando entran en contacto con los hijos sin perder de vista la trasmisión de afectividad y vínculo filial.

Lo previamente descrito, genera la forma en que el niño o la niña adquiere cogniciones interpretativas desde su círculo familiar y las extrapola a las interacciones con terceros, que en explicación de Figueroa (2009) les permiten hacer una comprensión emocional y la posterior entrega de una respuesta emocional hacia otros.

Otro de los factores causales hallados fue una baja auto cualificación de sí mismos, es decir un autoconcepto peyorativo del yo, ello está ligado con los procesos adquisitivos de la autorregulación emocional, según Quintero y Leiva (2015) maduran juntamente con las redes atencionales luego de los dos años a través del juego de manera activa cuya interacción le genera una mayor conciencia de sí mismo. 
El juego con los otros dentro de unas interacciones de satisfacción, frustración, ira, tolerancia y disfrute son esenciales, para López (2010), el juego es el espacio cuando se aleja de su realidad e inicia los esfuerzos por encontrar una identidad de sí mismo y de cómo quiere ser, cuya regulación proviene del intercambio con los demás compañeros de juegos.

En efecto, el aprendizaje derivado del juego es de alto impacto para la maduración y regulación emocional, ya que es el momento donde experimente varias situaciones las cuales generan una variedad de emociones que se entrecruzan con los participantes del juego, pero, también en ese momento aparecen los aprendizajes observacionales extraídos de su entorno que se articulan en medio del intercambio comunicativo y emocional.

Cuando este ejercicio lúdico no ha sido un discurrir favorable no son beneficiosos para el desarrollo emocional (López, 2010), y por consiguiente su situación contextual no se ciñe a las pautas más sanas de interacción y no resulta como buen aprestamiento para desenvolverse en el contexto social en el cual se halla inmerso.

A lo anterior, se suma como causalidades el rechazo por parte de los compañeros en la escuela, una cuestión estrechamente relacionada con las experiencias de los juegos y el aprendizaje emocional en su núcleo familiar, el rechazo que siente en su familia es la misma interpretación que atribuye a las interacciones con sus compañeros, en medio de la posición que asume de resistencia a dejar pautas emocionales negativas durante las experiencias lúdicas.

Acorde con ese diagnóstico se consideró pertinente acoger las orientaciones de Bisquerra (2009), en cuanto a la capacidad de reflexionar acerca del orgullo y el deseo personal, la expresión de manera espontánea del amor hacia otras personas, objetos o cosas, y por último reconocimiento de la intimidad y el secreto.

El trabajo pedagógico de un mes sustentado en actividades lúdicas y en aprovechamiento de las fortalezas detectadas, tal es el caso de la amistad y la dedicación, se convirtieron para ellos en un tiempo esperado con agrado, tanto por la inclusión de recursos tecnológicos que les fueron muy llamativos como la oportunidad de jugar con los demás.

Una vez concluido el trabajo formativo de sus emociones y al ser evaluado a los ocho días de su finalización, mostró mejoras en la administración de una manera más encausada de las emociones, no obstante, igualmente se determinaron algunas situaciones persistentes.

El abordaje sobre el bloque de mejoras hace apertura con la disminución de la agresividad física y verbal, Ministerio de Educación Nacional (MEN) (2014) expone que estas manifesta- 
ciones son motivadas por una causalidad externa, la cual es susceptible de ser reinterpretada con la ayuda adecuada para cambiar la respuesta emocional, tal como aconteció durante las actividades lúdicas que gradualmente llevaron a los estudiantes al análisis reflexivo de sus acciones y con ello decrecer malas actitudes durante los juegos.

De otro lado, sobre esta temática de la agresión están Montoya, Giraldo, Arango, Forgiarini y García (2014), quienes hallaron en su investigación que la indebida administración de las emociones está asociada con las conductas de agresión que se hacen más severas dadas las particularidades culturales e históricas de Colombia.

Ese mismo ejercicio lúdico con un repensar sobre la cognición interpretativa asignada a determinadas acciones durante el juego fue favorable en lo referente a las ofensas contra compañeros, en especial por acuerdo de otras pautas de interacción y comunicación ante los desacuerdos, y su valoración constante sin designar nombres ni calificaciones desagradables. Es decir, que se ratifica lo aportado por Reyes (2008) sobre la transformación valorativa de las causalidades externas.

En cuanto al desempeño académico las mejoras se trasladan a una parte de las conductas disruptivas durante el desarrollo de clases, así la observación reporta una mayor capacidad de atención prestada ante el trabajo de los educadores lo que favorece la receptividad cognitiva y la responsabilidad, por lo anterior se trae a citación Montoya, Giraldo, Arango, Forgiarini y García (2014), ya que estos investigadores en su estudio de trabajo con las emociones de los niños y las niñas refieren que se tiene un efecto positivo en lo cognitivo, afirmación presente también en los planteamientos de Otalora (2010).

Anexo a lo hasta ahora descrito, como parte de los logros alcanzados se encuentra la integración, al respecto Posso, Sepúlveda, Navarro y Laguna (2015) reconocen en el juego un vehículo exitoso para el manejo de las emociones al servicio de la convivencia, efectivamente, la interacción lúdica permite habilidades para relegar la ira y dar paso a tolerar la frustración para luego entrar a conciliar entre ellos antes que dejar prevalecer el oposicionismo, producto final el sentimiento de compañerismo.

El mismo principio de Posso, Sepúlveda, Navarro y Laguna (2015), se enmarca lo referente a la diversión colectiva, el aprender a comunicar sus emociones e ideas en ese lapso del juego, este último que a su vez es crucial para contrarrestar el aislamiento y que se sume al grupo para participar de la diversión al tiempo que de los aprendizajes emocionales que se desprenden de dicha actividad. 
Contrario a la narrativa previa, se ubican las situaciones en las cuales persisten las manifestaciones emocionales desfavorables, la primera de ellas a considerar en el correspondiente listado de esta índole son las ofensas a compañeros por el uso de apodos, lo cual es un ataque a la autoestima del otro, que bien puede ser intrascendente como no, ya que los desarrollos emocionales son subjetivos y en tanto unos maduran y se fortalecen en ese proceso otros no.

De ahí que, Gutiérrez, Fontenla, Cons, Rodríguez y Pazos (2017) hagan énfasis en que la educación emocional no puede estar limitada a indicar un aprendizaje donde se identifican las emociones entre negativas y positivas, sino que se requiere vivenciar las emociones y optar por las más convenientes para regular las cargas emocionales; en especial porque la autoestima está vinculada estrechamente con la posibilidad del individuo para vivir de forma sana y con equilibrio sus emociones.

Así mismo, se observó persistencia del autoritarismo reflejado en la imposición de la opinión propia ante los compañeros, este tipo de posturas según Sánchez y Quiceno (2016) están relacionadas con la formación familiar que pondera el yo sin considerar que la vida no es de islas con habitantes únicos, sino de existir al lado de muchos otros, por eso no se puede viabilizar el que sea una sola identidad la que asuma debe prevalecer, lo que es exaltar el individualismo y las dificultades que sobrevienen con esa línea de actuación.

La falta de este tipo de regulación emocional también afecta la formación de valores como es el irrespeto, ya que definir y nominar a las personas por apodos es despojar a los otros de consideración y una buena valoración, por tal motivo es que se considera una falta en los manuales de convivencia de las instituciones educativas.

Otra problemática resistente para ser superada fue el rechazo, una situación que se presenta ante la falta de Comprensión Emocional (CE), de acuerdo con Nóbrega y Franco (2014), la CE es un componente de alta importancia en la formación de inteligencia emocional ya que es precisamente ella la que conduce al sentimiento de afinidad y la posibilidad de seleccionar a alguien con fines relacionales, es decir que CE es precisamente la encargada en incrementar la aceptación del otro, en consecuencia evitar el que algunos rechacen a otros.

Nóbrega y Franco (2014), afirman que si se quiere derribar las situaciones de rechazo como una condición de exclusión es imprescindible que la formación en CE inicie desde edades tempranas como el preescolar y así viabilizar la adquisición de este tipo de competencia emocional con mayor arraigo en los individuos, y por lógica dar cabida a una mejor fluidez en las relaciones entre congéneres. 
En este mismo sentido se hallan las divisiones entre niños y niñas, cuando se excluye al uno o al otro de las actividades ya sean cuando se proponen jugar o en cualquier otro momento para departir en grupo, una actitud que entre los adultos lleva a la segregación e incluso a la eliminación del diferente, razón por la cual resulta necesario dotar a los estudiantes desde los inicios de sus procesos formativos donde la CE sea una práctica constante con una inteligencia emocional que no cree brechas entre géneros para tener momento de gratificación mutua en actividades diversas a lo largo de la vida.

La falta de CE entre compañeros en los escenarios escolares y que debilita la aceptación de los demás, refuerza la timidez de quienes poseen una tendencia a ser introvertidos y poco expresivos de sus ideas o emociones, el que existan niños o niñas que rechazan a los otros, explica la postura continuista de quienes en su rol de estudiantes son tímidos.

Finalmente, sobre las dificultades no superadas en lo que corresponde al desempeño escolar, algunos de los participantes se aferran a la indisciplina con notaria afectación para la parte atencional durante el proceso de enseñanza aprendizaje, y sin una respuesta positiva a los llamados de atención ante los cuales muestran desobediencia en un abierto desacato a las normas.

Entonces, con base en lo expuesto sobre los resultados arrojados por el proceso investigativo, especialmente, los hallazgos posteriores al trabajo de educación emocional, es válido afirmar que su aplicación genero logros, que si bien es cierto no favorecieron la superación total de las problemáticas iniciales, si arrojaron impacto de encause a varias emociones y producto de ello transformaciones en las conductas de convivencia y en la motivación escolar como lo exponen Garner y Waajid (2008).

\section{CONCLUSIONES}

El nivel de aprendizaje emocional para la convivencia escolar entre los estudiantes de preescolar y primero de básica primaria de la Institución Educativa Pacarní del municipio de Tesalia, reside en diferentes prácticas que obstruyen la convivencia pacífica, reconociendo estas como: el maltrato a compañeros, conductas disruptivas, actitudes de indisciplina, imposición de opinión, el oposicionismo y diversas reacciones burlescas con sus compañeros aunado al trato verbal soez, así como la tendencia a aislarse. No obstante, hay prácticas que potencian esta convivencia como algunos niños que promueven la amistad y la dedicación.

En cuanto a los factores que generan estas conductas ligadas a las emociones entre los estudiantes se puede destacar como relevante el sentir que no compaginan dentro de su círculo 
familiar y consigo mismo, teniendo en cuenta que algunos compañeros los excluyen de sus actividades escolares, provocando comportamientos como: distracciones dentro de la clase, burlas y el uso de lenguaje soez a los compañeros.

Por ende, se reflexionó sobre el uso de estrategias lúdicas, las cuales, tuvieron un proceso diagnóstico, implicando que se propusieran actividades mediante el uso de material participativo, multimedia y objetos virtuales de aprendizaje, estas se relacionaban con el reconocimiento de las emociones, toleración a la frustración, empatía, control de la ira, intimidad y secreto, manejo de agresividad y las habilidades de cortesía social.

Los efectos de las estrategias lúdicas aumentaron la participación en algunos estudiantes, debido a que generaron lazos de confianza e integraron con los otros compañeros, así mismo, adquirieron habilidades de responsabilidad para realizar la actividad, mostrándose receptivos frente a las actividades de multimedia y los objetos virtuales de aprendizaje. Respecto a la convivencia hubo comportamientos que se pueden clasificar como fallas, es decir, se reprodujo: el individualismo, irrespeto, rechazo, imposición de opinión y, escisión de niños y niñas; aunque se generaron construcciones de convivencia positiva como es la participación, comunicación de sus cosas, compañerismo y receptividad.

En suma, las diferentes actividades lúdicas propuestas generan que el desempeño escolar aumente, ya que promueven el trabajo colectivo, crece el interés por las metodologías utilizadas y los estudiantes ponen atención a lo enseñado, por lo cual, se construye unos comportamientos dentro del aula que generan unos matices de sana convivencia.

\section{LIMITACIONES DEL ESTUDIO}

El actual trabajo sobre la educación de la inteligencia emocional en edades tempranas de niños escolarizados responde a característica específicas de una población en una zona rural en la región sur de Colombia. Ello implica que, si bien es cierto que sus contenidos pueden ser aprovechados, es preciso adelantar ajustes que permitan una atención en respuesta certera a una realidad en particular.

Igualmente, es de señalar que se trabajó con una parte de la población escolarizada de los grados de preescolar y primero de primaria, como una estrategia pedagógica, sin que ello esté asociado a una situación de índole curricular con sus respectivos componentes para el abordaje académico dentro de un periodo lectivo. La innovación se limita a indicar el impacto derivado de la estrategia que tuvo por tiempo de implementación un mes, en especial porque 
sobre la sociedad global se cernía la amenaza de una pandemia y la inminente decisión estatal de confinamiento y aislamiento social, lo que llevo a tomar la decisión de adelantar el trabajo en solo un mes para no asumir un riesgo que obstaculizar la ejecución del mismo.

Debido a lo descrito con antelación, sería pertinente dar a esta base investigativa una continuidad de trabajo pedagógico durante un tiempo mayor, de tal forma que esa condición sea susceptible de valorar en cuanto a incremento del impacto de la estrategia o no.

\section{REFERENCIAS BIBLIOGRÁFICAS}

Ayers, S., Hamada, H. \& Wyatt, T. (2011). Factor Structure of Self-Regulation in Preschoolers: Testing Models of a Field-Based Assessment for Predicting Early School Readiness. Journal of Experimental Child Psychology, 111(3), p.386-404. [DOI: 10.1016 / j.jecp.2011.10.002]

Ayers, S., Hamada, H. \& Zinsser, K. (2012). Early Childhood Teachers as Socializers of Young Children's Emotional Competence. Early Childhood Education Journal 40(3), p.137-143. [DOI: 10.1007 / s10643-012-0504-2]

Bisquerra, R. (2009). Psicopedagogía de las emociones. Editorial Síntesis, Madrid.

Carbajal, Y. Flores, K., Polo, J. \& Torres, C. (2018). Inteligencia emocional y desempeño docente en las instituciones educativas FAP de Lima. Trabajo de grado para el título de Master en Educación, Universidad Marcelino Champagnat. [http://repositorio.umch.edu.pe/bitstream/ UMCH/544/1/66.\%20Tesis\%20\%28Flores\%20Daorta\%2c\%20Polo\%20Churrango\%20y\%20Torres\%20Agui$\underline{\text { lar\%29.pdf] }}$

Cefai, C. \& Cavioni, V. (2014). Social and emotional education in primary school: integrating theory and research into practice. NY, USA: Springer.

Figueroa, L. (2009). Sobre la educación de los sentimientos. Tiempo de educar, año 10, segunda época, número 19, enero-junio, p.77-111. [https://www.redalyc.org/pdf/311/31113164004.pdf]

Gardner, H. (1983). Estructuras de la mente-La teoría de las inteligencias múltiples. New York: Harper Collins Publishers.

Garner, P.W. \& Waajid, B. (2008) The associations of emotion knowledge and teacher-child relationships to preschool children's school-related developmental competence. Journal of Applied Developmental Psychology, 29, p.89-100. [https://doi.org/10.1016/j.appdev.2007.12.001]

Goleman, D. (1995). Inteligencia Emocional. Editorial Kairos, México. 
Gutiérrez, L.; Fontenla, E.; Cons, M.; Rodríguez, J.E.; Pazos, J. Mª (2017). Mejora de la autoestima e inteligencia emocional a través de la psicomotricidad y de talleres de habilidades sociales. Sportis Sci J, 3 (1), p.703-721. [DOI: 10.17979/sportis.2017.3.1.1813]

Henao, G. y García, M. (2009). Interacción familiar y desarrollo emocional en niños y niñas. Revista latinoamericana de ciencia, sociedad, niñez y juventud, Vol.7, No.2, p.785-802. [https://www. redalyc.org/articulo.oa?id=77315614009]

Hernández; R., Fernández, C. \& Baptista, P. (2014). Metodología de la Investigación. (6ed.) México: Mc Graw Hill.

Hermans, B. \& Collins, R. (2018). Competencias del aprendizaje social y emocional. Departamento de Instrucción Pública de Wisconsin

López, I. (2010). El juego en la educación infantil y primaria. Revista autodidacta, consultada en noviembre 30 de 2019 y disponible en: http://educacioninicial.mx/wp-content/uploads/2017/11/JuegoEIP.pdf

Marchant, T., Milicic, N. \& Álamos, P. (2013). Impacto en los niños de un programa de desarrollo socio-emocional en dos colegios vulnerables en Chile. Revista Iberoamericana de Evaluación Educativa, 6(2), p.167-186. [https://revistas.uam.es/riee/article/view/3411/3628]

Martínez, J. (2015). Relación entre Inteligencia emocional y autoestima en niños de 5 años. Trabajo para optar al título de Máster en Neuropsicología y Educación, de la Universidad Internacional de La Rioja. [https://reunir.unir.net/bitstream/handle/123456789/3715/MARTINEZ\%20CELIS\%2c\%20 JUAN\%20NICOLAS.pdf?sequence $=1$ \&isAllowed $=\mathrm{y}$ ]

Ministerio de Educación Nacional (2014). Guías pedagógicas para la convivencia escolar. Guía No.49. Información disponible en: https://aprende.colombiaaprende.edu.co/sites/default/files/naspublic/ Guia\%20No.\%2049.pdf

Montoya, D., Giraldo, N., Arango, L., Forgiarini, R. \& García, A. (2014). Características cognitivas, emocionales y conductuales De niños preescolares del programa buen comienzo en el Noroccidente de Medellín. AGO.USB, Medellín-Colombia, V. 14 No 2, p. 311- 703. [http://www.scielo. org.co/pdf/agor/v14n2/v14n2a15.pdf]

Nóbrega, N. \& Franco, G. (2014). Inteligencia emocional: la comprensión emocional y las relaciones entre pares en el jardín de infancia. INFAD Revista de Psicología, №1-Vol.5, p.167-176. [https:// www.redalyc.org/pdf/3498/349851788018.pdf]

Otalora, Y. (2010). Diseño de espacios educativos significativos para el desarrollo de competencias en la infancia. Indagaciones y Perspectivas en Psicología Social No.5, p. 71-96. [http://www.scielo. org.co/pdf/recs/n5/n5a04.pdf] 
Peña, D.L. (2013). Ambiente de aprendizaje lúdico: una experiencia en el fortalecimiento de valores en niños de segundo año de preescolar. Trabajo para optar al título de Licenciada en Educación Preescolar, Universidad de Morelos, México.

Posso, P., Sepúlveda, M., Navarro, N. \& Laguna, C.E. (2015) La lúdica como estrategia pedagógica para fortalecer la convivencia escolar. Lúdica Pedagógica, (21), p.163-164.[ DOI: 10.17227/01214128.21ludica163.174]

Quintero, L. \& Leiva, M. (2015). Desarrollo emocional y afectivo en la primera infancia "Un mundo por descubrir y formar en los infantes”. Monografía presentada como requisito para obtener el grado de psicóloga, Universidad Nacional Abierta y a Distancia -UNAD-, Neiva (Huila), zona sur, Colombia.

Reyes, F. (2008). Inteligencia emocional proceso enseñanza-aprendizaje educación preescolar. Trabajo de grado para optar al título de Licenciada en Educación, Universidad Pedagógica Nacional de México.

Sánchez, A. \& Quiceno, J. (2016). Significados de convivencia: una comprensión desde la dinámica cotidiana que los promueve. Pensando Psicología, 12(19), p.55-67. [DOI: 10.16925/pe.v12i19.1328]

Silva, R. \& Calvo, S. (2014). La actividad infantil y el desarrollo emocional en la infancia. Revista Intercontinental de Psicología y Educación, vol. 16, núm. 2, julio-diciembre, p. 9-30. [https://www. redalyc.org/pdf/802/80231541002.pdf]

Temis, C., Wau, N. \& Hughes, D. (2007). Objetivos de Padres de Niños: La coexistencia dinámica de individualismo y colectivismo en culturas e individuos. Blackwell Publishing Ltda.

UNESCO (2011). Una Crisis encubierta: conflictos armados y educación; Informe de seguimiento de la EPT en el mundo. En: https://unesdoc.unesco.org/ark:/48223/pf0000192155 [Fecha de consulta: noviembre 28 de 2019].

Whitebread, D. \& Basilio, M. (2012). Emergencia y desarrollo temprano de la autorregulación en niños preescolares. Profesorado, revista de curriculum y formación de profesor, Vol.16, No.1, p. 1434. [http://www.ugr.es/ recfpro/rev161ART2.pdf] 